\title{
The effects of varying the interreinforcement interval on appetitive contextual conditioning
}

\author{
ALBA E. MUSTACA and FABIAN GABELLI \\ Universidad de Buenos Aires, Buenos Aires, Argentina \\ MAURICIO R. PAPINI \\ Texas Christian University, Fort Worth, Texas \\ and \\ PETER BALSAM \\ Barnard College of Columbia University, New York, New York
}

\begin{abstract}
Appetitive contextual conditioning in rats and ringdoves was investigated in six experiments. In Experiment 1, differential contextual training produced greater anticipatory activity in rats in the presence of a context paired with food than it did in rats in the presence of a different context in which food was never presented. Furthermore, the rats showed a preference for the context associated with food when they were given a simultaneous choice test between contexts. In Experiment 2, rats were more active in and preferred a context associated with a variabletime 30-sec (VT30) schedule as opposed to a VT180 schedule. Experiment 3 was a between-subjects replication of the previous experiment. As expected, rats exhibited significantly more anticipatory activity in a context in which food had been presented on a VT30 schedule than they did in a context in which food had been presented on a VT180 schedule. Experiment 4 showed that anticipatory activity was a reflection of context-US associations in ringdoves, and in Experiments 5 and 6 , ringdoves also exhibited an inverse relationship between the amount of anticipatory activity and the length of the interreinforcement interval (IRI). These results reveal a relation between IRI and contextual conditioning opposite from that obtained in studies of aversive conditioning.
\end{abstract}

Temporal variables exert a strong influence on associative learning (Gibbon \& Balsam, 1981; Gormezano \& Kehoe, 1981). Perhaps the best known example is the trial-spacing effect, in which acquisition of an association between a conditioned stimulus (CS) and an unconditioned stimulus (US) is facilitated by lengthening the intertrial intervals (ITIs; see Gormezano \& Moore, 1969). Several different underlying mechanisms have been proposed to account for the trial-spacing effect.

Variation in ITI may influence acquisition by affecting US processing or effectiveness. The repeated presentation of the US may result in habituation, and since there is greater short-term habituation with shorter intervals between stimuli (Davis, 1970), the US may be relatively less effective when trials are massed rather than more widely spaced. Yet even as this general prediction follows from all theories of habituation, more specific predic-

We thank J. Deich, M. Fanselow, and J. Gibbon for comments on earlier versions of this manuscript. Experiments 1-3 were carried out at the University of Buenos Aires and supported by the Consejo Nacional de Investigaciones Cientificas y Tecnicas, Argentina. Experiments 4-6 were conducted at Barnard College and supported by NIMH Grant RO1 34759. Correspondence should be addressed to either P. Balsam, Psychology Department, Barnard College, Columbia University, New York, NY 10027, or M. R. Papini, Psychology Department, Texas Christian University, Fort Worth, TX 76129. tions can be derived from particular theories about how the effectiveness of a stimulus changes with repeated presentations. Solomon and Corbit (1974) suggest that reception of a US generates two opponent processes. For example, an electric shock releases a short-latency, rapid-decay primary process (the a process) of an aversive nature. The primary process gives rise to a long-latency, slow-decay secondary process (the $b$ process) that opposes the aversive state. The effective motivational state of the subject is a function of the difference between the two processes. Since the secondary process is slow to decay, when ITIs are relatively short, the secondary process induced on trial $n-1$ may decrease the motivational properties of the US presented on trial $n$. Assuming that the rapid-decay primary process does not cumulate across trials, the net effect of massed trial presentation will be to render the US less effective. Longer ITIs, as opposed to massed trials, will provide an opportunity for the secondary process to decay more prior to subsequent US presentation.

In a similar vein, Wagner's (1978) rehearsal model suggests that short ITIs produce slower acquisition because the processing of the CS and/or US on trial $n-1$ may extend until trial $n$, thus attenuating the processing of these events relative to what would take place when trials are more widely spaced. This is based on the model's assumption that processing is increased by a surprising event and decreased when the representation of an event is 
moved into an active state by its recent presentation (i.e., self-generated priming). Since the formation of associations is a function of the amount of time that the CS and US are simultaneously rehearsed, massed US presentations will result in less conditioning than will spaced US presentations.

More recently, Wagner (1981; Wagner \& Larew, 1985) has formalized these assumptions about CS and US processing in the SOP model. In this view, presentation of an event causes a representation of that event to move into a primary state (A1). This representation decays into a secondary state (A2), and finally, with the passage of time, back into an inactive state. Excitatory associations are formed when the CS and US representations are simultaneously in the primary state, and inhibitory connections are formed when the CS is in the primary state and the US is in the secondary state. Thus, when trials are closely spaced, the US representation might still be in the secondary state from previous activation. Consequently, massed trials might generate some inhibitory conditioning by activating primary processing of the CS while secondary processing of the US is taking place. In addition, activation of the US representation is attenuated if it is already in the secondary state, rendering it less likely to enter into excitatory associations. Therefore, this model posits two means whereby massed training could result in less net excitatory strength than spaced training would (Ewing, Larew, \& Wagner, 1985).

Alternatively, trial spacing effects might be mediated by conditioning of the context. For example, the model of Rescorla and Wagner (1972; henceforth referred to as the Rescorla-Wagner model) assumes that there is competition between cues and contexts for associative value. Nonreinforced exposure to the contextual cues during the ITI are assumed to extinguish the associative value of the context, so that long ITIs produce more extinction than briefer ITIs do. Consequently, the facilitated performance under spaced conditions is the result of relatively little interference by contextual stimuli with the CSs acquisition of associative value. Alternatively, massed trials might produce greater conditioning of the context but influence the performance controlled by the CS rather than the learning about the signal. In their application of scalar expectancy theory to autoshaping, Gibbon and Balsam (1981) suggested that the expectancies controlled by the CS and the context are inversely related to their respective durations. Response strength is assumed to be determined by the ratio of these expectancies. When trials are widely spaced, background expectancy will be low relative to trial expectancy. When trials are massed, the expectancy of the US in the presence of contextual stimuli may be sufficiently high to interfere with responding to the CS, even though the expectancy controlled by the signal may be the same in both massed and spaced conditions. Similarly, Miller and his associates (Miller \& Matzel, 1988; Miller \& Schachtman, 1985) have suggested that response strength is determined by a comparison of the current associative strength of the signal with the current associative strength of the context in which that signal was trained. Responding to the signal will emerge only to the extent that its associative strength is higher than that of the training context.

Rather than the context being the cue that competes with the CS for associative value, Goddard and Jenkins (1988) have suggested that one US may come to predict subsequent USs. If this learning is mediated by some aftereffect of US presentation, it might be expected that such a cue would be more salient after a short period of time than it would after a longer delay. Thus, to the extent that a US will act as a signal for subsequent USs and interfere with learning about other simultaneously presented cues, we might anticipate less conditioning of the CS and context with massed as opposed to spaced training.

Although these theories agree when they predict that acquisition of conditioned responding to a discrete CS is enhanced by relatively long ITIs, they produce divergent predictions for the situation in which no explicit signal precedes the US. On the one hand, the Rescorla-Wagner model and the model of Gibbon and Balsam (1981; henceforth referred to as the Gibbon-Balsam model) predict that the context will gain more associative strength when USs are massed than it will when USs are spaced. In the Rescorla-Wagner theory, briefer periods of nonreinforced exposure to the context-alone, when USs are massed, result in higher asymptotic levels of context-US association than they do when USs are more widely spaced. According to Gibbon and Balsam (1981), asymptotic context expectancies are inversely related to the average time between US presentations. Thus, both theories predict that the strength of contextual conditioning will be directly related to US frequency.

On the other hand, Wagner's models and the opponentprocess theory predict no better, and possibly worse, contextual conditioning as a result of massed training. Repeated US presentations may produce a decrement in US effectiveness caused by self-generated priming (Wagner, 1978), presentation of the US while the secondary state is still present (Wagner, 1981; Wagner \& Larew, 1985), or recruitment of the opponent process (Solomon \& Corbit, 1974). Since massed US presentations may produce a greater decrement in US effectiveness than spaced US presentations, contextual conditioning might be diminished under the former conditions. Similarly, Goddard and Jenkins's (1988) findings suggest that massed training might enhance the ability of the US to become a signal for the next US and to thus overshadow the context, relative to spaced training.

Available evidence derived from aversive conditioning preparations seems to support Wagner's $(1978,1981)$ models and the opponent-process theory. In several experiments, relatively long interreinforcement intervals (IRIs) have enhanced the development of contextual conditioning. Bolles and Riley (1973), for example, delivered electric shocks to rat subjects at IRI values of 10,43 , or 
$900 \mathrm{sec}$ and found an increasing amount of freezing as the IRI was increased. The experiment does not, however, allow for a clear assessment of whether this was a conditioned or unconditioned effect of shock presentation, because freezing was only recorded during training sessions.

McAllister, McAllister, Weldin, and Cohen (1974) trained several groups of rats in an aversive conditioning situation in which unsignaled shocks were delivered at different IRIs. In a nonreinforced test carried out a day later, the rats could escape from the conditioning chamber by jumping into a neutral box; the latency to escape was used as a measure of conditioned fear in response to contextual cues. With IRIs values of 165,225 , and $285 \mathrm{sec}$, escape latencies were shortest after exposure to shocks presented with the longest IRI.

More recently, Fanselow and Tighe (1988) replicated the effect of IRI length on the freezing behavior of the rat by using a procedure similar to that of Bolles and Riley (1973), except that testing was carried out after $24 \mathrm{~h}$, thus eliminating any unconditioned effects of recent US presentations. Interestingly, they found an IRI effect after delivering only two USs, the minimum number of shocks necessary to give rise to the IRI dimension. In fact, they obtained more freezing after two shocks presented with an IRI of $60 \mathrm{sec}$ than they obtained after 16 shocks presented with an IRI equal to $3 \mathrm{sec}$.

Data from appetitive conditioning experiments suggest that one might obtain a different relationship between IRI and context conditioning from that observed with aversive USs. Using speed of conditioning as a measure of prior contextual conditioning, Balsam, Locurto, Terrace, and Gibbon (1980) found no systematic relation between the duration of the IRI during US-only pretraining and subsequent speed of autoshaping in pigeons. Tomie and Abbondandolo (1981), however, found that pigeons pretrained with short IRIs tended to acquire subsequent keypecking more slowly than did pigeons pretrained with longer IRIs; but the effect failed to reach statistical significance. Killeen $(1975,1979)$ found that general activity levels in pigeons were directly related to reinforcer frequency, but in these experiments it is not possible to distinguish between the unconditioned activity elicited by the prior US presentation and activity conditioned to the context that occurs in anticipation of the impending US presentation. Hence, there is some, albeit weak, evidence that unlike aversive conditioning, the strength of contextUS associations may be inversely related to IRI duration in appetitive contextual conditioning.

The present experiments were aimed at investigating the effect of IRI duration on appetitive contextual conditioning with both rats (Experiments 2 and 3 ) and ringdoves as subjects (Experiments 5 and 6). Experiments 1 and 4 validate the testing techniques in rats and ringdoves, respectively. Conditioning to contextual stimuli was assessed on preference tests in which subjects chose between distinctive contexts associated with different reinforcement rates and/or by measuring conditioned activity following exposure to different IRIs.

\section{EXPERIMENT 1}

In pigeons and rats, conditioned anticipatory activity has been observed in the presence of cues paired with food (Longo, Klempay, \& Bitterman, 1964; Sheffield \& Campbell, 1954; Zamble, 1967), and, in the case of pigeons and doves, general activity increases in the presence of contextual cues associated with food presentation (Balsam, 1982, 1985; Durlach, 1982, 1983). However, there is little information about appetitive contextual conditioning in the rat. Thus it was necessary first to develop ways of measuring context-US associations in this species.

In experiments with fixed-time (FT) schedules of unsignaled reinforcer presentation, rats typically show increments in a variety of activities with food or water USs, both after and before US delivery, including goal-centered activity (Reid, Vazquez, \& Rico, 1985; Staddon \& Ayres, 1975 ) and a variety of interim activities such as gnawing, drinking, or wheel running, for which there is environmental support (Riley, Wetherington, Delamater, Peele, \& Dacanay, 1985; Staddon, 1977). Although experiments with FT schedules may provide some clues to which responses may be appropriate indices of contextual learning in rats, they are not definitive on this point. The increase in activity may be attributed to conditioning effects such as the superstitious conditioning of whatever behaviors precede the reinforcer (Skinner, 1948), Pavlovian temporal conditioning (Pavlov, 1927), or the formation of context-US associations (Balsam, 1982, 1985; Durlach, 1983). Alternatively, the increased activity may be a direct consequence of US presentations. Responses may be directly elicited by the US, and repeated US presentations have been shown to increase a variety of scheduleinduced or adjunctive behaviors (Falk, 1971; Staddon, 1977), elicited by discriminable periods of low reinforcement probability (Minor, 1987; Staddon \& Simmelhag, 1971), and/or the cumulation of elicited arousal across trials (Killeen, Hanson, \& Osborne, 1979). However, in the usual situations in which these activities have been observed (during training sessions), one cannot distinguish between activities that are elicited by the preceding food presentation and activities that occur in anticipation of the next one. In order to determine whether or not any of these actions are conditioned, the behavior must be observed in the absence of US presentations, and it must be shown that the responses only occur in the presence of cues that have been present at the time of US presentation.

Thus we initially measured a wide range of behaviors in the presence of contextual cues. In addition, we measured the preference (cf. Balsam, 1985) for contexts differentially associated with food. Preference for a particular context after appetitive conditioning would presumably reflect a greater anticipation of food in the preferred context than in a less preferred context. Our specific purpose 
in Experiment 1 was to validate these measures of appetitive contextual conditioning by comparing a context in which food had previously been presented and a context in which food had never been presented.

\section{Method}

Subjects. Eight female, 90-day-old rats (CHBB TOM Strain) from one litter were used as subjects. Their ad-lib weights varied between 200 and $260 \mathrm{~g}$. The animals were experimentally naive, lived in individual cages, and were exposed to a 12:12-h light:dark cycle (light from 0800 to $2000 \mathrm{~h}$ ). The temperature was maintained at $20^{\circ} \mathrm{C}$.

Apparatus. The arms of a Y-maze served as conditioning contexts. During conditioning sessions, doors were placed in the entrance of each arm to confine subjects to a particular context. During preference tests, doors were raised so that the animals could move from one arm to the other through the choice area of the maze. The internal dimensions of each context arm were $79 \times 40 \times 60 \mathrm{~cm}$. The choice area was $34 \mathrm{~cm}$ long. The maze was built with wood and fully lined with formica. The walls were covered with white formica, and the floors were covered with light-blue formica. The ceiling was made of glass. A small Plexiglas container was located at the end of each arm, illuminated by a single bulb $(15 \mathrm{~W})$ placed approximately $25 \mathrm{~cm}$ above each container. Reinforcers (Purina rat chow pellets of $0.2 \mathrm{~g}$ each) were manually delivered through a tube into the appropriate container. Masking white noise $(70 \mathrm{~dB}, \mathrm{SPL})$ was presented through speakers located above the food container. The experimental contexts were differentiated by the stimuli on the walls and floor of the arms of the $Y$-maze. One context had a smooth formica floor and a mirror $(50 \times 39 \mathrm{~cm})$ mounted on one of the sidewalls of the arm. The second context had a metallic grid $(48.5 \times 23 \mathrm{~cm}$, with $0.5-\mathrm{cm}$-diam holes) on the floor of the arm adjacent to the food container and had smooth formica walls.

The maze was located in a sound-attenuated room. Temperature was maintained at $22^{\circ} \mathrm{C}$ throughout the experiment.

Procedure. Access to food was limited to 2-h daily periods, starting 10 days before the initiation of training and continuing throughout the experiment. This procedure leads to a stable weight of $80 \%-85 \%$ of the ad-lib weight for each subject (Hurwitz \& Davis, 1983). Water was continuously available in the cages. Pretraining consisted of two 10-min sessions (one in each arm) in which the animals were placed in the conditioning box without the elements that were later used to differentiate contexts (i.e., the mirror and the metallic grid). They received 10 USs in a variable-time 60 -sec (VT60) schedule.

Before the start of conditioning, animals received a preference pretest that comprised two 5-min sessions in which they could freely move from one arm of the maze to the other. The two sessions were conducted on the same day and separated by at least $90 \mathrm{~min}$. The stimuli that differentiated contexts were present during the preference test. Context element (mirror and grid) and position (right and left arm) were counterbalanced across sessions. During each preference test, animals were placed in the choice area; a digital clock was started whenever the animal introduced its four legs into an arm, and it was stopped when the animal placed its four legs on the choice area. Total time spent in each arm was recorded.

Beginning on the day after the pretest, the animals were exposed to a differential conditioning procedure. During the initial 10 days, they received one session per day in which they were exposed to one of the contexts (i.e., $\mathrm{X}+$ and $\mathrm{Y}-$ ) in random order, except that the first session was $X+$ for all the animals. Each conditioning session began with a 5-min period in which no USs were presented. During the remainder of rewarded sessions, 10 USs were presented on a VT60 schedule. Exposure to the negative context consisted of confinement to the appropriate arm of the maze for a comparable period of time, but no USs were presented during the entire ses- sion. The particular stimuli that defined the positive and negative contexts were counterbalanced across subjects, and the spatial location of the contexts (whether each context appeared as the left or the right arm of the maze) was counterbalanced across sessions.

During the initial 5-min nonreinforced portion of each session, an instantaneous sampling of behavior was carried out every $15 \mathrm{sec}$ by observing the animal for approximately $1 \mathrm{sec}$. Observations involved recording both the location of the subject and the specific behavior that was displayed. Location was recorded as being in one of two places: (1) door, when the animal was located between the door and a line demarcating the start of the contextual element (either the mirror or the metallic grid, both of which were about $30 \mathrm{~cm}$ from the door); and (2) element, when the animal was located with four legs within the limits of the contextual element. Five behavior categories were also scored: (1) Activity was recorded when movements of all four limbs were observed during the sampling period (this included instances of walking, running, circling, or jumping); (2) feeder was scored when the rat was sniffing at, biting, or introducing its head into the feeder, or rearing in front of the feeder; (3) rear was scored when the animal was standing on its hindlimbs with forelimbs not on the floor; (4) grooming was scored when the rat displayed licking of any part of its body; and (5) still was scored when there was a complete absence of movement, with all limbs on the floor. In each sampling period, the observer recorded the location (door or element) and the behaviors (activity, feeder, rear, grooming, and still).

The first preference test was carried out on Day 11. This test was identical to the pretest described above. From Days 12-21, animals received another series of conditioning sessions with the same characteristics that were described above for the initial conditioning sessions. Finally, a second preference test was carried out on Day 22.

\section{Results and Discussion}

All animals ate the food delivered during pretraining sessions. The pretest of preference indicated that subjects spent 71.7 and $41.5 \mathrm{sec}$ in the arms that were subsequently to become the $\mathrm{X}+$ and $\mathrm{Y}-$ contexts, respectively. The mean difference was attributable to 2 subjects who showed a strong preference for the arm that was to become the $\mathrm{X}+$. There was no statistically significant difference between groups in the amount of time spent in each arm during the pretest. Data from the two postacquisition preference tests were analyzed in a test session (first or second) $\times$ context (positive or negative) analysis of variance (ANOVA). Overall, the animals spent significantly more time in the context associated with food than they did in the context in which food had never been presented $[F(1,7)=17.83, p<.01]$. Since the test $\times$ context interaction was also significant $[F(1,7)=17.03, p<.01]$, pairwise tests were calculated to determine the source of the interaction. After the first 10 conditioning sessions, the animals spent more time in the context associated with food $(M=86 \mathrm{sec})$ than they did in the context in which food had never been presented $(M=68.7 \mathrm{sec})$, but this difference was only marginally significant $[t(7)=2.01$, $.05<p<.10$ ]. At the end of training, the animals spent significantly $[t(7)=5.72, p<.01]$ more time in the positive context $(M=126.2 \mathrm{sec})$ than they did in the negative context $(M=77.2 \mathrm{sec})$.

Activity was the only behavior that yielded a consistent pattern across sessions and contexts. These results 


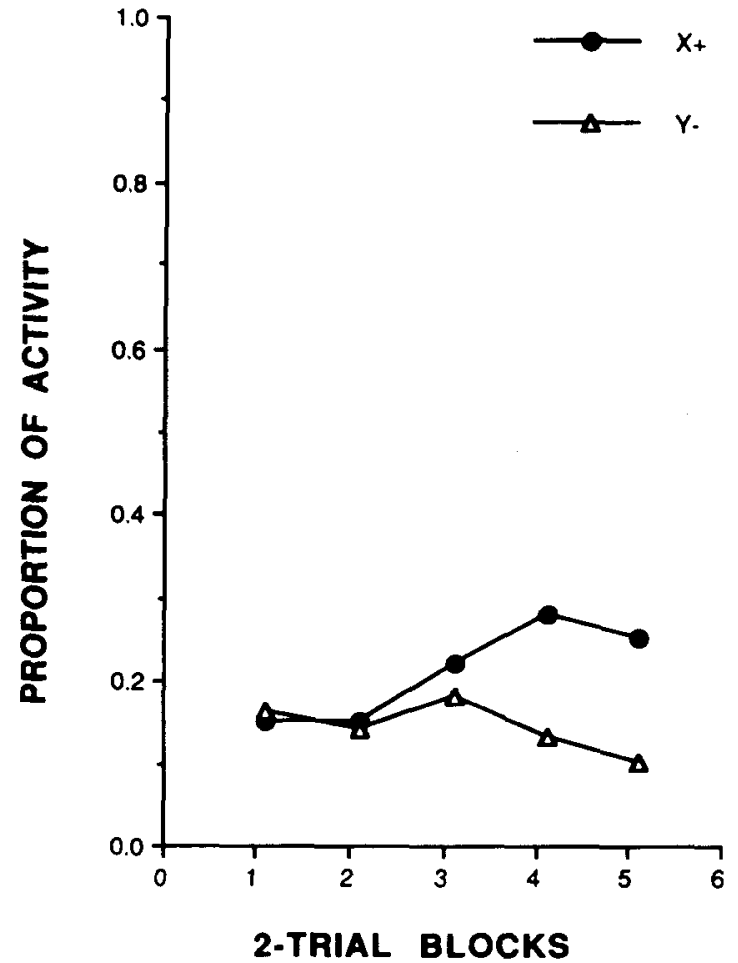

Figure 1. Mean proportion of intervals in which subjects were active in the reinforced $(X+)$ and nonreinforced $(Y-)$ contexts.

are plotted in Figure 1 in terms of the mean proportion of sampling periods during the 5-min observation period at the beginning of each session in which activity was scored. A context (positive or negative) $\times$ blocks (of two sessions) ANOV A (both factors with repeated measures) indicated that the animals showed significantly more activity in $\mathrm{X}+$ than they did in $\mathrm{Y}-[F(1,7)=10.63$, $p<.025]$. The context $\times$ blocks interaction was also significant $[F(4,28)=4.35, p<.01]$. Tests of simple main effects indicated that only in Blocks 4 and 5 did subjects exhibit significantly more activity in the positive context than they did in the negative one.

These results showed that rats discriminated between two contexts that were differentially associated with food, exhibiting both a preference to remain in the arm containing the contextual element previously paired with food, and a higher level of activity in that context. The basis for these differences between contexts would appear to be the result of associative processes, since the withinsubjects design, coupled with the contextual specificity of behavioral changes, makes a nonassociative account unlikely. Furthermore, the counterbalancing of contextual elements with respect to their relation to reinforcement and their position precludes possible unconditioned effects of the stimulus elements themselves. Thus both preference and activity level would appear to be measures of context-US associations in the rat.

The increase in general activity in anticipation of food presentation is similar to the behavioral changes that have been observed in pigeons (Durlach, 1982, 1983) and ringdoves (Balsam, 1982, 1985) under similar circumstances. This contrasts with the goal-centered responses observed in red opossums (Papini, Mustaca, Tiscornia, \& Di Tella, 1987). One reason for the differences between these experiments may be the nature of the US that was employed. General activity occurs as the CR to solid food USs, whereas goal-centered behavior rather than general activity reflects context-US associations when a liquid US is employed (Papini, Mustaca, \& Frasca Ponce, 1989).

One unanticipated aspect of the current results was the relatively slow acquisition rate. Prior work has shown that contextual learning can be relatively rapid (e.g., Balsam \& Gibbon, 1988; Balsam \& Schwartz, 1981; Durlach, 1983). Several methodological aspects of the procedure might account for the slow acquisition speed obtained in this experiment, including: reinforced pretraining sessions; the use of a 5-min sampling period, which might have produced substantial contextual extinction since it involved one third of the session length; and the use of a food container, which might have made the two contexts more similar and also might have competed with contextual elements for associative value. Finally, the elements used to differentiate the boxes (mirror and metallic grid) may not have been particularly salient.

\section{EXPERIMENT 2}

Experiment 1 provided information about specific behavioral changes that occur as a consequence of pairing a set of contextual stimuli with the delivery of food. Experiment 2 was designed to investigate whether variation in the IRI will produce differences in contextual conditioning. Preference for particular contexts and anticipatory activity were used to assay differences in the strength of context-US associations. Animals were exposed to different VT schedules in each context: VT30 versus VT 180. Experiment 2, therefore, represents a test of models that predict better contextual conditioning under massed conditions (e.g., Gibbon \& Balsam, 1981; Rescorla \& Wagner, 1972) as opposed to models that predict better contextual learning under spaced conditions (e.g., Solomon \& Corbit, 1974; Wagner, 1978).

The present experiment was similar to Experiment 1, except for some changes in procedure designed to facilitate discriminative conditioning. The pretraining consisted of nonreinforced exposure to the contextual elements (instead of nondifferential reinforcement); behavioral sampling was carried out during the initial $90 \mathrm{sec}$ (instead of $5 \mathrm{~min}$ ) of only four sessions (instead of each session); and the food container was eliminated, so that pellets were scattered about the floor.

\section{Method}

Subjects and Apparatus. Twelve experimentally naive female rats, of the same strain, age, and weight as in the previous experiment, served as subjects. Maintenance conditions were identical to those described above.

The apparatus was the same as that used in Experiment 1, with the following modifications. The food container was eliminated so 
that pellets fell onto the floor. The two contexts were distinguished by the materials on the floors and walls. One context had two wood plates $(66 \times 20 \mathrm{~cm})$, with black (2-cm-wide) and white (4-cm-wide) vertical stripes located in the two sidewalls and in the light-blue formica floor. The second context had white formica walls and an aluminum plate $(59 \times 32 \mathrm{~cm})$ placed on the floor.

Procedure. The animals received one 5-min unreinforced preexposure session in the presence of each of the contexts on each of two consecutive days. The order of presentation and position of each element in each of the maze's arms were counterbalanced across subjects. The second preexposure session in each context was preceded by $90 \mathrm{sec}$ of behavioral sampling. Activity was recorded at sampling intervals of $10 \mathrm{sec}$, following the technique described in Experiment 1.

Conditioning involved 20 daily sessions. In half of the sessions, food was presented on a VT30 schedule (range, 11-46 sec), and in the remaining sessions, food was presented on a VT180 schedule (range, 69-279 sec). In a given day, half of the animals were trained under VT30 and half under VT180. For half of the animals, the vertical stripes were paired with the VT30 schedule, and the aluminum plate was paired with the VT180 schedule; for the rest, the relationship was the opposite. The order of conditions across sessions was pseudorandom, with the restriction that a given context was not presented for more than two successive sessions. Ten pellets of rat chow $(0.2 \mathrm{~g}$ each) were delivered in each session. During the initial $90 \mathrm{sec}$ of Sessions 9, 10, 19, and 20 (two sessions for each VT condition), two independent observers recorded activity according to the definition and sampling procedure described for Experiment 1. An interobserver index of agreement was calculated by dividing the smaller frequency of activity scored by one observer over the larger one, for each animal and session. An average score was then calculated for each animal, and then a general mean of this index was computed for all animals. This value was 0.92 . Finally, on the day following the last training session, the animals were given a preference test identical to those in Experiment 1 .

\section{Results and Discussion}

By the third session, most of the animals ate the pellets immediately after food delivery. One animal failed to eat all of the pellets immediately after delivery in Session 5, and a second animal failed to eat all of the pellets immediately during Sessions 5,7 , and 9 . Nevertheless, their scores were included in all the analyses.

During the pretest, activity levels were similar in both contexts (mean proportion of intervals with activity for VT30 and V'T 180 contexts were 0.51 and 0.46 , respectively). After five sessions of exposure to each VT schedule, the animals were exhibiting a higher proportion of activity in the context associated with the shorter IRI, and the difference was replicated in the data collected prior to the last training session in each context. These results are presented in Figure 2 in terms of the proportion of sampling periods (out of a maximum of 9) in which activity was scored. An IRI $\times$ sampling session ANOVA (both factors with repeated measures) indicated that activity was significantly higher in VT30 than it was in VT180 $[F(1,11)=17.62, p<.01]$. There was no significant effect of sampling session, nor was there a significant IRI $\times$ sampling session interaction.

The results of the preference test that followed the final conditioning session showed that the animals spent significantly $[F(1,11)=10.96, p<.01]$ more time in the con-

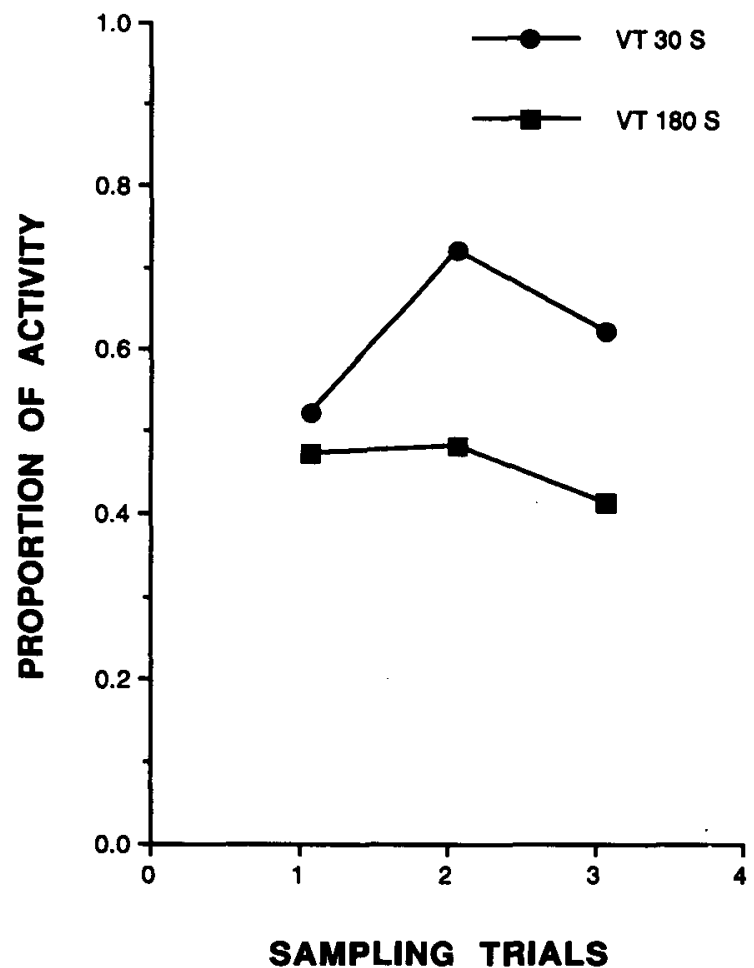

Figure 2. Mean proportion of sampling periods in which subjects were active in contexts associated with either a VT30 or a VT180 schedule.

text associated with the VT30 $(M=149.7 \mathrm{sec})$ than they did in the context associated with the VT180 schedule $(M=48.6 \mathrm{sec})$.

A greater difference between contexts emerged in Experiment 2 than had been observed in Experiment 1, which suggests that some or all the modifications introduced were effective in facilitating discriminative conditioning. The asymptotic level of activity in each context appears to have been reached in under 40 US presentations. This pattern of results suggests that appetitive contextual conditioning can occur relatively rapidly for the rat, as it does for pigeons (Balsam \& Gibbon, 1988; Durlach, 1983) and ringdoves (Balsam \& Schwartz, 1981).

Experiment 2 showed that rats exhibit greater anticipatory activity in the presence of a context associated with a short IRI than in the presence of contextual cues associated with more widely spaced food presentations. In addition, when given a choice between contexts associated with different rates of food presentation, the rats consistently preferred the one associated with the more frequent food presentation. Thus it appears that the strength of context-US associations is greater after massed US presentation than it is after spaced US presentation.

The results of this experiment support the prediction derived from both the Gibbon-Balsam and the RescorlaWagner models-that shorter IRIs produce a higher asymptotic strength of the appropriate contextual elements. This 
provides indirect support for the theories in which it is supposed that trial spacing effects are mediated by contextCS interactions. Specifically, these data give credence to the hypothesis that the associative strength of the context may either block the learning of discrete CSs (Rescorla \& Wagner, 1972) or interfere with the control of performance by discrete CSs (Gibbon \& Balsam, 1981) under relatively short, as compared to long, ITIs (e.g., Gibbon, Baldock, Locurto, Gold, \& Terrace, 1977; Rescorla \& Durlach, 1987).

These results are inconsistent with Wagner's rehearsal model and the opponent-process theory, both of which predict facilitated conditioning with longer IRIs. In addition, the present data disagree with previous experiments on the effects of the IRI in the acquisition of contextual conditioning (Bolles \& Riley, 1973; Fanselow \& Tighe, 1988; McAllister et al., 1974). There are some potentially important procedural differences between these experiments, but the robustness of the effects reported in the literature suggests that an important parameter may be the type of reinforcer used (i.e., aversive vs. appetitive).

\section{EXPERIMENT 3}

An unusual feature of Experiment 2 as opposed to previous studies on the effect of IRI is the use of a withinsubjects design. Such designs may be more sensitive, because in addition to differences in associative strength, they are sensitive to potential contrast effects that may enhance the phenomenon. If the results of the previous experiment depend on the use of a within-subjects design, the generality of the influence of IRI on context may be severely limited (see Holder \& Roberts, 1988). Therefore, it was relevant to determine the extent to which the effect of IRI would be the same when independent groups trained with different schedules were compared. In addition, this experiment differed from the previous ones in two other ways. First, we used rats from a different strain (Sprague-Dawley) and sex to determine the extent to which the effect of the IRI on anticipatory activity was peculiar to these features in the previous experiment. Second, we used different contextual cues in independent groups to determine whether acquisition speed depends to some extent on the availability of multiple contexts of different associative value.

\section{Method}

Subjects and Apparatus. Twenty-four rats (derived from Sprague-Dawley strains), all males, 90 days old, and experimentally naive, served as subjects. Housing and deprivation conditions were as in Experiment 2. The conditioning boxes and the contextual cues were those used in Experiment 2 . The only difference was that each animal was exposed to only one box; therefore, for half of the animals, the box contained the vertical black and white stripes on the walls and the light-blue floor, whereas for the remaining animals, the walls were white and the floor was covered by the aluminum plate.

Procedure. Pretraining consisted of a 5-min session of exposure to the conditioning box, without the contextual elements (vertical stripes on the walls or metallic plate on the floor) and without USs.
Conditioning began the day after pretraining and lasted for 15 daily sessions. In each session, animals received 10 pellets of rat chow $(0.2 \mathrm{~g}$ each). In Group $30(n=12)$, the 10 pellets were delivered according to a VT30 schedule. In Group $180(n=12)$, pellets were provided according to a VT180 schedule. Half of the animals in each group were trained in each context. Unfortunately, 2 animals trained with VT30 in the context with the aluminum plate had to be dropped from the experiment because of illness. Using observation procedures identical to those in the previous experiments, activity during the initial $100 \mathrm{sec}$ of Sessions $1,5,10$, and 15 was recorded. No USs were delivered during the observation period.

\section{Results and Discussion}

All of the subjects consumed the pellets immediately after presentation by the end of the first session. Rats tended to exhibit more activity when food was cued by the aluminum plate, but since this effect occurred from the first sampling session, it appears to be an unconditioned effect of this stimulus upon performance. The main results of the experiment are presented in Figure 3, where data from both contexts were pooled to show only the effect of IRI. The figure shows the proportion of sampling periods in which activity was scored as a function of the VT schedule, during each of the four sessions in which behavioral samplings were carried out.

An IRI $\times$ context $\times$ session ANOVA indicated that there was a significant main effect of IRI. The animals trained on the VT30 schedule displayed significantly more anticipatory activity than did those trained under the VT180

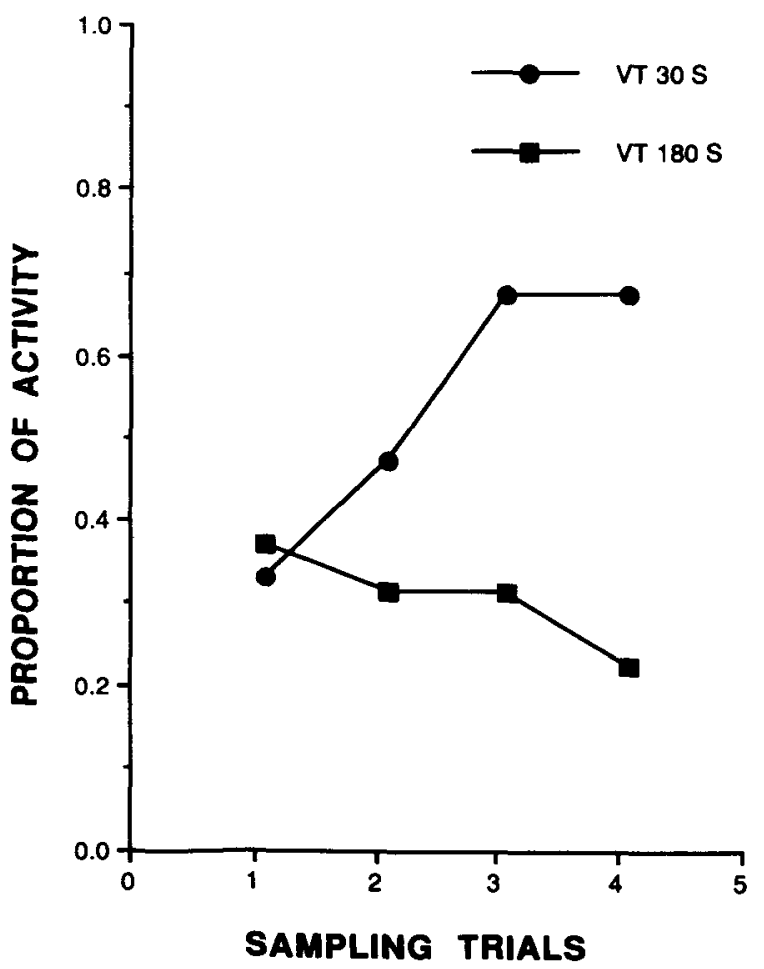

Figure 3. Mean proportion of sampling periods in which subjects were active in contexts associated with either a VT30 or a VT180 schedule. 
schedule $[F(1,18)=23.67, p<.01]$. There was also a significant effect of context $[F(1,18)=5.73, p<.05]$ on activity. The increasing difference between groups over sessions was reflected in a significant $\mathbf{I R I} \times$ sessions interaction $[F(3,54)=7.11, p<.01]$. No other interactions were significant.

As in the previous experiment, the current results indicate that there is greater appetitive contextual conditioning after exposure to massed US presentation than there is after more widely spaced food presentation. The use of a between-subjects design shows that this effect is not dependent on a contrast between reinforcement conditions in the training situation. Hence, the differences between the current results and those that have been reported for aversive conditioning (see introduction) do not seem attributable to the use of a particular type of experimental design. In addition, the presence of the same pattern of results in two different strains of rats and both sexes (in Experiments 2 and 3 ) suggests that the difference between aversive and appetitive conditioning is also not likely to be due to subject differences.

\section{EXPERIMENT 4}

The discrepancy between prior experimental results involving the effects of IRI in aversive contextual conditioning and the present results suggests that it may be worthwhile to determine the generality of the effect for the appetitive case. In the next two experiments, we employed a training situation that was very different from that used in the previous studies. First, the subjects were ringdoves instead of rats. Second, we looked at conditioned activity in extinction as well as before and during conditioning sessions. Experiment 4 validated the use of general locomotor activity as an index of contextual conditioning, and in Experiments 5 and 6, we examined the effect of varying the IRI on conditioned and unconditioned activity.

In Experiment 4, we employed a between-subjects design to investigate whether or not unsignaled US presentations result in conditioned locomotor activity in ringdoves. Two groups of subjects were given food presentations followed by nonreinforced exposure to contextual cues that were either the same as or different from the cues that were present during the food presentations. If the activity induced by food presentation is a conditioned behavior, then subjects tested in the presence of the pretrained cues should be more active than subjects tested in a novel context. Furthermore, like other conditioned responses, the contextual CRs should decline during extinction and show spontaneous recovery across sessions.

\section{Method}

Subjects. Twelve experimentally naive ringdoves (Streptopelia roseogrisea) maintained at $85 \%$ of their free-feeding weights (120-150 g) served as subjects.

Apparatus. Four identical Coleman ice chests were converted into conditioning chambers with internal dimensions of $33 \times 30 \times 26 \mathrm{~cm}$. All of the walls were painted with a flat-black enamel. Though not used in the current experiment, one response key, $2.5 \mathrm{~cm}$ in di- ameter was located $12 \mathrm{~cm}$ above the floor and $8 \mathrm{~cm}$ to the left of the midline of the intelligence panel. The floor consisted of a $2 \times 3$ array of $14 \times 9.5 \mathrm{~cm}$ movable panels. Each panel was hinged along the narrow dimension to one wall (either to the intelligence panel or to the parallel wall) and rested on a microswitch. Approximately $85 \mathrm{~g}$ of weight in the center of each panel caused a switch closure. White noise ( $80 \mathrm{~dB}$, SPL in front of the feeder) could be presented through a speaker mounted behind the intelligence panel. General illumination was provided by four No. 1829 bulbs mounted at the top of the panel. There was a $6.5 \times 6.5 \mathrm{~cm}$ hopper aperture $3 \mathrm{~cm}$ above the floor and centered in the intelligence panel. The hopper was equipped with a photocell used to sense the entrance of a bird's head into the aperture.

A cardboard shell that could be inserted into each chamber was used to create visually distinct contexts. Each shell had a cutout for the response key and for the feeder opening. The inside of the shell was covered with vinyl contact paper that had a floral print on a white background. When the shell was in place, a low level of ambient illumination inside the chamber provided whatever light from the houselights penetrated the shell and entered through the cutouts, and the white noise was turned off. Thus there were two physical contexts: one was the bright, noisy, black-walled chamber; the other was the dim, quiet, lined chamber.

Procedure. All birds were exposed to 25 daily sessions in which 20 feeder presentations were initiated on an FT36 schedule. The first session began with the feeder in the raised position and some grain resting on the lip of the aperture. All subsequent sessions began with a 36-sec period signaled by the onset of the houselights. All feeder presentations were timed from the moment when the subject interrupted the photocell and lasted for $4 \mathrm{sec}$. Half of the subjects were trained in the bright, noisy, black context, and the remainder of the subjects were trained in the dim, quiet, lined context. All birds were then exposed to five context extinction sessions consisting of $720 \mathrm{sec}$ of nonreinforced exposure to the experimental chamber. For half of the birds, extinction sessions occurred in the context that had been present during the previous training phase. The remaining birds were given nonreinforced exposure to the context that they had not yet experienced. In the next phase of the experiment, the birds were given 4 additional context extinction sessions in the alternate context. Thus, following conditioning, all birds experienced 9 sessions of nonreinforced exposure to the experimental chamber. Half of the birds (Group Same-Diff) experienced 5 of these sessions in their training context, followed by 4 sessions in a novel context. The remaining birds (Group Diff-Same) experienced 5 sessions in the novel context, followed by 4 sessions in their training context.

Activity scores consisted of the accumulated closures of the floor switches as the dove moved around the experimental chamber. The dependent variable in the current studies consisted of these activity scores for the period prior to US presentation at the start of each session and the activity recorded during extinction sessions.

\section{Results and Discussion}

During feeder training, all birds quickly learned to eat from the feeder. Within two sessions, all birds were eating with an average latency of less than $2.7 \mathrm{sec}$.

All birds showed considerable levels of activity during the initial phase of Experiment 4. The mean level of activity during the last five training sessions was 14.54 responses/min for the group tested in their training context during Phase 2, and it was 15.89 responses/min for the group subsequently tested in the novel context. The mean level of activity during the beginning of these sessions (prior to any US presentation) was equal to 11.08 responses/min and 15.19 responses/min for the Phase 2 


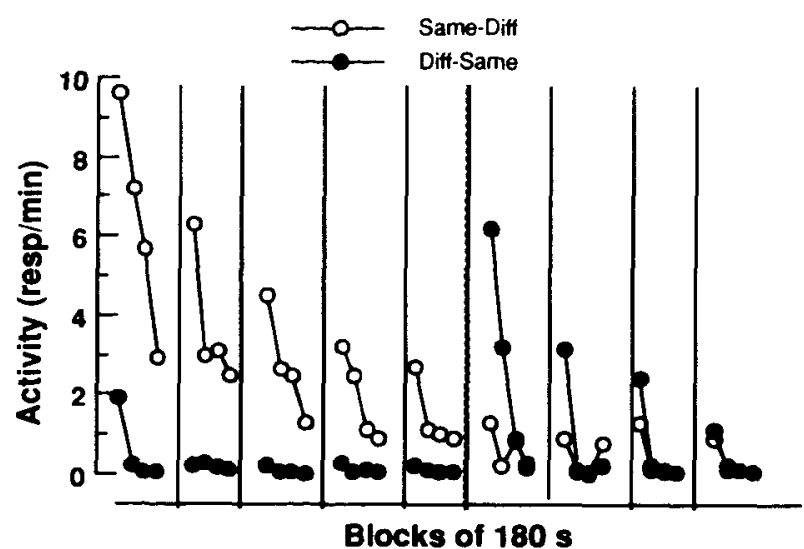

Figure 4. Mean activity rate during nonreinforced exposure to the context is shown in session quartiles. Each session is separated by the vertical lines. Group 1 was first tested in the training context and then tested in a different context, as shown before and after the hatched line. Group 2 was first tested in a context different from the training context and then returned to the training context for the last four sessions.

groups tested in the training and novel contexts, respectively. During this first phase of the experiment, there was no statistical difference between these groups in either the total activity in training sessions or the activity prior to the first US presentation $[F \mathrm{~s}(1,10)<1.9, p>.05]$.

The mean level of activity during extinction sessions is shown in Figure 4. Each session is divided into quartiles (180-sec periods). The activity levels in each phase of extinction testing were analyzed separately in a groups $x$ sessions $x$ blocks ANOVA. During the first extinction test, the birds tested in the training context (Group SameDiff) were significantly more active than the birds tested in the novel context (Group Diff-Same) $[F(1,10)=5.89$, $p<.05]$. Activity decreased across sessions $[F(4,40)=$ $7.07, p<.01]$ and across blocks within a session $[F(3,30)$ $=10.67, p<.011$. The birds tested in the training context also showed spontaneous recovery from the end of 1 day to the beginning of the next. The attenuated difference between groups with increasing nonreinforced exposure to the contexts was reflected in significant groups $\times$ sessions $[F(4,40)=4.83, p<.01]$ and groups $X$ blocks $[F(3,30)=4.87, p<.01]$ interactions. No other interaction was significant. A test of simple main effects showed that the groups differed significantly from each other on Days 1,2 , and $5[F \mathrm{~s}(1,10)>5.0, p<.05]$.

Data from the second phase of extinction testing showed a similar pattern of results. Again, the birds returned to their training context (Group Diff-Same) responded at significantly higher levels than did the birds tested in the novel context (Group Same-Diff) $[F(1,10)=4.93, p<$ $.05]$. Activity decreased over sessions $[F(3,30)=10.17$, $p<.01]$ and blocks $[F(3,30)=42.71, p<.01]$. The significant groups $\times$ blocks $[F(3,30)=15.42, p<.01]$ and groups $\times$ sessions $[F(3,30)=5.12, p<.01]$ inter- actions reflected the attenuated difference between groups within and across sessions. The attenuation of spontaneous recovery across sessions was reflected in a significant sessions $\times$ blocks interaction $[F(9,90)=3.22, p<$ .01]. Finally, the groups $\times$ sessions $\times$ blocks interaction was also significant $[F(9,90)=3.53, p<.01]$. Tests of simple main effects showed that there was a significant difference between groups only on the 1st day of this phase $[F(1,10)=8.17, p<.05]$.

As in the previous experiments with rats, a substantial amount of conditioned locomotor activity occurred as a result of unsignaled food presentation. Four findings in Experiment 4 argue that a large portion of this activity is, in fact, a CR controlled by contextual cues previously paired with food. First, substantial activity occurred prior to reinforcer presentation in each training session. Second, like other conditioned responses, this activity declined as a result of nonreinforced exposure to the CS (context). Third, the response showed spontaneous recovery from the end of one extinction session to the beginning of the next session. Finally, the context switch manipulation showed that this activity occurred only in the presence of contextual cues previously paired with the US. Thus, activity controlled by the context exhibits many of the usual properties of a CR.

\section{EXPERIMENT 5}

In the previous experiments with rats, it was found that there was an inverse relationship between conditioned activity and the duration of the IRI. In Experiment 5, we examined this relationship in ringdoves by using the methods developed in Experiment 4.

\section{Method}

Subjects and Apparatus. Nine experimentally naive ringdoves maintained at $85 \%$ of their free-feeding weights $(120-150 \mathrm{~g})$ served as subjects. The apparatus was identical to the unlined chamber used in Experiment 4.

Procedure. As in Experiment 4, the first session began with the feeder in the raised position and some grain resting on the lip of the aperture. Feeder presentations were timed from when the subject interrupted the photocell and lasted for $4 \mathrm{sec}$. The birds were exposed to daily sessions in which 20 feeder presentations were initiated on one of three FT schedules: FT36, FT72, or FT144. Each subject was exposed to all FT values, and the order of exposure to schedules was counterbalanced across subjects. Each FT schedule was in effect for 12 consecutive sessions.

\section{Results and Discussion}

All birds quickly ate from the hopper within the first session. As in Experiment 4, the birds showed a great deal of activity throughout conditioning sessions at all IRIs. The activity rate prior to presentation of the first US is shown in Figure 5, averaged across the last five sessions of exposure to each of the IRIs. A one-way, repeated measures ANOVA showed a significant effect of IRI on anticipatory activity $[F(2,16)=5.75, p<.05]$. Pairwise comparisons showed that subjects were significantly 


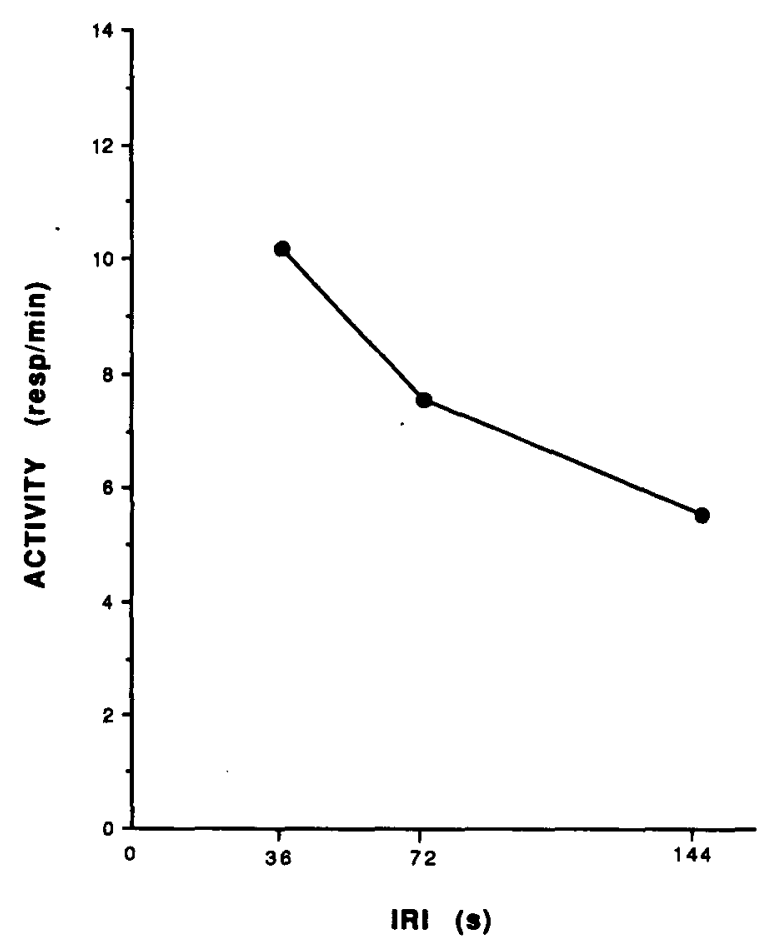

Figure 5. Mean activity rate during the last five sessions of exposure to each of the interreinforcement intervals. The pre-US rate represents the activity recorded at the beginning of a session prior to US presentation.

more active at the 36- and 72-sec IRIs than they were at the $144-\mathrm{sec}$ IRI $[t \mathrm{~s}(8)=3.04$ and 2.47 , respectively; ps $<.05]$.

These results replicate the inverse relation between IRI and strength of context conditioning found in the studies with rats (Experiments 2 and 3). These findings are inconsistent with theories that posit increases in US processing or effectiveness with increases in the IRI (Solomon \& Corbit, 1974; Wagner, 1978, 1981), and they are contrary to the previously described empirical relationship found in aversive conditioning.

In addition, the results of Experiment 5 show that one needs to be cautious in interpreting the results of studies on the effects of varying the IRI on interim or adjunctive behavior in which subjects are exposed to repeated US presentations. In those studies, it is generally not possible to tell whether the measured behaviors were elicited by the previous reinforcer or conditioned responses in anticipation of impending reward. For example, our finding that substantial activity is conditioned to contextual cues and that the amount of activity is inversely related to the IRI runs counter to previous conceptualizations of this activity in terms of the cumulation of unconditioned arousal (Killeen et al., 1979). More generally, these results highlight the difficulties of inferring the underlying cause of behavior from its time of occurrence in the IRI (Falk, 1971; Staddon, 1977). It seems worthwhile to investigate the source of control for other schedule-induced, adjunctive, or interim behaviors in designs that permit the differentiation of conditioned and unconditioned behavior (see, e.g., Minor, 1987).

\section{EXPERIMENT 6}

In the current experiments, an inverse relationship between conditioned activity and the duration of the IRI was obtained in contrast to the opposite relation that has been described for aversive USs. Although it is possible that there may be different rules for appetitive and aversive learning, there are also enough procedural differences between our experiments and studies of aversive control that they cannot be ruled out as important factors in producing the differing results. For example, the range of IRIs studied in both the aversive and the appetitive cases has not been that extensive. If there was a bitonic relationship between IRI and contextual conditioning, researchers in the aversive studies may have been studying one arm of this function, whereas in our appetitive studies, we may have inadvertently examined the other arm of the function.

It is indeed difficult to imagine that contextual conditioning in the aversive case would increase monotonically as the IRI is increased. At the extreme, the nonreinforced period of the IRI should be long enough to result in substantial extinction. Some empirical support for this hypothesis comes from studies of unsignaled avoidance. To the extent that unsignaled avoidance procedures are mediated by contextual fear, subjects do prefer longer times between shocks to briefer times between shocks (Hineline, 1977; Sidman, 1962). In addition, there is some evidence that no freezing will occur when shocks are very widely spaced (Fanselow \& Lester, 1988). Thus it is possible that spacing aversive USs does decrease contextual conditioning over some range of IRIs. In the appetitive case, perhaps, IRIs briefer than the ones we employed in the previous experiments would result in diminished appetitive contextual conditioning.

The purpose of Experiment 6 was to investigate this possibility by exposing subjects to very brief IRIs, well within the range of IRIs studied with aversive USs. Ringdoves were exposed to IRIs of 6 and $36 \mathrm{sec}$, and conditioned activity was measured both prior to the start of each session and during extinction sessions following training sessions at each IRI.

\section{Method}

Subjects and Apparatus. Eight ringdoves (Streptopelia roseogrisea) maintained at $85 \%$ of their free-feeding weights served as subjects. These birds had previous experience in autoshaping experiments with keylight CSs and grain USs. The apparatus was identical to the unlined chamber used in the previous experiment.

Procedure. The birds were first exposed to two 20-min background extinction sessions during which no USs were presented. After these two sessions, all birds showed very low activity rates. Subsequently, the birds were exposed to daily sessions in which 20 4-sec feeder presentations were initiated on either an FT6 or an FT36 schedule. Each session began with a 100 -sec period during which no USs were presented. Each bird was exposed to both FT values, and the order of exposure to schedules was counter- 
balanced across subjects. Each FT schedule was in effect for four consecutive sessions. Following exposure to each of the FT schedules, the birds were given one 20 -min session of nonreinforced exposure to the experimental chamber.

\section{Results and Discussion}

All subjects quickly ate from the hopper within the first session. As in Experiment 5, the birds showed a great deal of activity throughout conditioning sessions at both IRIs. The mean activity rate during the extinction sessions following exposure to each of the IRIs is shown in Figure 6. All birds were more active in the extinction session following exposure to the shorter IRI. A repeated measures $t$ test showed that following exposure to the 6-sec IRI, the birds were significantly more active than they were following exposure to the 36-sec IRI $[t(7)=3.53, p<$ $.01]$. An additional measure of conditioned activity was obtained through examination of the average activity rate prior to US presentation during the last sessions of exposure to each of the IRIs. One subject did not move at all at the start of its session under either IRI. Nevertheless, the mean activity rates in anticipation of the first US were 8.48 and 4.65 responses/min for the 6- and $36-\mathrm{sec}$ conditions, respectively. Because of the inactive subject, there was only a borderline significant difference between conditions on this measure $[t(7)=2.34, p<.06]$. If that subject is excluded from the analysis, the statistic reaches conventional significance levels $[t(6)=2.46, p<.05]$.

These results again replicate the inverse relation between IRI and strength of context conditioning found in

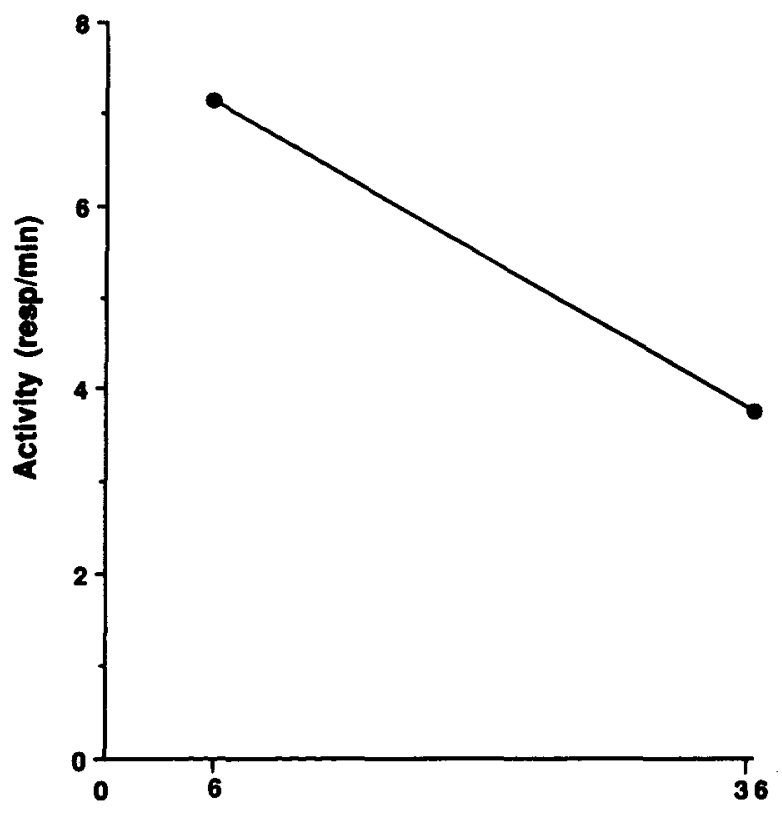

IRI (s)

Figure 6. Mean activity rate during the background extinction session following each of the interreinforcement intervals. our previous studies. Hence, there is no evidence of a bitonic relationship between IRI and appetitive contextual conditioning. Although it is possible that we have not sampled the appropriate IRIs for detecting a bitonic function, we think it more likely that we have found a reliable difference between the appetitive and aversive cases. The possible reasons for such differences are discussed in the next section.

\section{GENERAL DISCUSSION}

The present series of experiments strongly supports the hypothesis that when appetitive USs are presented with short IRIs there is greater contextual conditioning than is observed after exposure to longer IRIs. This relationship was found in ringdoves and in two strains of rats. It was observed both in terms of anticipatory activity levels elicited in the presence of the contextual stimuli previously paired with food, and in terms of a preference for contexts associated with higher rates of food presentation. It was found in between- and within-subjects designs, showing that the effect is context-specific. Finally, it was found to be reversible; animals exposed to a short FT schedule could later develop an activity level appropriate to a longer FT schedule, and vice versa.

In contrast to the typical deleterious effects of massed practice upon learning about a signal, contextual learning benefited from the massed delivery of food. These results are consistent with the Rescorla-Wagner and Gibbon-Balsam models, both of which predict greater conditioning of contextual cues paired with more frequent USs. This finding is also relevant to an understanding of the above-mentioned effects of massed training on the formation of CS-US associations. These models claim that response deficits after massed CS-US training are caused by the strengthening of context-US associations, although, as noted earlier, the mechanisms postulated as underlying this effect are different. Nevertheless, to the extent that the current experiments confirm that widely spaced USs produce less context conditioning than closely spaced USs do, they support the hypothesis that contextual conditioning might mediate the trial spacing effect (Gibbon et al., 1977; Rescorla \& Durlach, 1987).

Our results appear to be inconsistent with other models that anticipate the strength of contextual conditioning produced by unsignaled US presentation to be at least as great with spaced training as with massed training (Solomon \& Corbit, 1974; Wagner, 1978, 1981). However, the specific application of these models to particular experiments allows for a great deal of flexibility in predictions. Specific predictions depend on the temporal dynamics of the particular response system being studied. For example, if food USs induce a relatively long-lasting and strong primary state and a relatively brief and weak secondary state, massed training might result in cumulation of the primary state and augmented conditioning. Furthermore, Wagner's (1981) analysis allows for the conditioned primary and secondary states to be either antagonistic or 
synergistic. Thus the particular parameters of an experiment will interact with the dynamic and motivational properties of a particular response system and may produce outcomes ranging from facilitation to interference as a result of massing US presentations. It is therefore possible to account for both the effects of food spacing found in the current experiments and the exact opposite results of studies employing aversive USs (Bolles \& Riley, 1973; Fanselow \& Tighe, 1988; McAllister et al., 1974).

The dynamic theories suggest two accounts for why food and shock USs might produce different trial-spacing effects. First, shocks may induce an opponent state that reduces US effectiveness, whereas food may not. Evidence (Fanselow, 1991; Fanselow \& Bolles, 1979) does suggest that shock presentations trigger an endogenous analgesic mechanism. A shock-induced analgesic process could serve to reduce the functional magnitude of the aversive US. In addition, this analgesic effect may have a conditioned component (Fanselow, 1981, 1984; Grau, 1987; Lysle \& Fowler, 1988; Ross \& Randich, 1985) that attenuates conditioned fear during testing. Along these lines, Fanselow (1981) has shown that when rats are given the opioid antagonist naloxone, there is an increment in aversive contextual conditioning. Thus the effects of IRI on aversive contextual conditioning may reflect an interaction of the conditioned analgesic response with the conditioned fear response. If the analgesic response is somehow more strongly conditioned at short IRIs, one might find less fear exhibited after training with short IRIs as opposed to long IRIs. Although it is uncertain that massed US presentation does in fact serve to evoke a greater opponent process, increases in shock intensity do increase the analgesic effect (Fanselow \& Bolles, 1979; Hayes, Bennett, Newlon, \& Mayer, 1978). Perhaps the use of relatively mild shocks during training or the use of naloxone during training and/or testing would more clearly reveal the effects of IRI on the strength of context-US associations. On the other hand, food USs might produce a conditioned or unconditioned secondary state that augments the primary state, resulting in greater excitatory responding after massed US presentations. Thus the differences between food and shock USs might be the result of differences in the processing and/or motivational dynamics of the primary and secondary states educed by these stimuli.

Though possible, this is an unlikely account of the present results. Kaplan (1984) found that in an explicitly unpaired procedure with intervals from US to CS comparable to our US-US interval, pigeons showed good inhibitory conditioning. Thus the dynamic properties of the feeding system would seem to require that the secondary state be active at the time of US presentation and that the secondary state be antagonistic to the primary state. It should be noted, however, that Kaplan used pigeons as subjects, whereas in the present experiments we employed ringdoves and rats.

There is no a priori reason to assume that there is a divergence in learning principles produced by the use of hedonically different USs; as far as is known, aversive learning follows the same principles as appetitive learning does (Mackintosh, 1974, 1983), although there may be parametric differences. Thus an account of the behavioral divergence such as the preceding one has much appeal. However, there are still enough procedural differences between the experiments for one to be cautious about invoking a difference in the underlying processes as the reason for the discrepancy in findings.

First, as mentioned earlier, there is perhaps a bitonic function between IRI and context conditioning; through bad fortune, we may have failed to choose IRI values that would reveal this relation. Second, since we held the number of USs per session constant in each of our experiments, IRI was confounded with total time in the context. However, it is unclear how this might account for the difference between results in appetitive and aversive situations. Third, in all of the aversive experiments a single training day has been used, whereas in our experiments, we have used many US presentations over multiple days. Perhaps length of training and/or number of USs per session are crucial modulators of the relation between IRI and context conditioning.

Another difference between the aversive and the appetitive experiments lies in the dependent measures used in each case. It is possible that regardless of the nature of the US, massed presentations induce more general activity than spaced presentations do. If one measures general activity, the results of the current appetitive experiments would be obtained. On the other hand, if one measures the periods of time in which there is no movement (freezing), the opposite relation would result. Thus the apparent difference between the appetitive and aversive results may stem from a difference in measurement and not a difference in underlying process.

Finally, if the results of the aversive conditioning experiments do reflect conditioned fear, it is possible that the specific responses that are induced may change as a function of the IRI. Fanselow (Fanselow, 1989; Fanselow \& Lester, 1988) has suggested that a dimension of predatory imminence underlies response selection in aversive Pavlovian conditioning. In this view, CR topographies may change as a function of the imminence of the aversive stimulus. Thus Fanselow (1989) acknowledges that conditioned fear may be stronger with short IRIs than with long IRIs, but rats may be less likely to manifest that fear by freezing when a shock is quite imminent.

In summary, we have shown that appetitive contextual conditioning is enhanced by relatively massed food presentation, a finding that is consistent with some models of Pavlovian conditioning (Gibbon \& Balsam, 1981; Rescorla \& Wagner, 1972) and presents some difficulties for other accounts based on US processing (Wagner, 1978, 1981 ) or opponent processes (Solomon \& Corbit, 1974). The fact that this relation is the opposite of that reported for aversive conditioning opens some important questions about the commonalities and differences between appetitive and aversive conditioning. 


\section{REFERENCES}

Balsam, P. (1982). Bringing the background to the foreground: The role of contextual cues in autoshaping. In $\mathbf{M}$. Commons, A. R. Wagner, \& R. J. Hermstein (Eds.), Quantitative analysis of behavior: Vol. 3. Acquisition (pp. 145-171). Cambridge, MA: Ballinger.

BALSAM, P. (1985). The functions of context in learning and performance. In P. Balsam \& A. Tomie (Eds.), Context and learning (pp. 121). Hillsdale, NJ: Erlbaum.

Balsam, P., Grbbon, J. (1988). Formation of tone-US associations does not interfere with the formation of context-US associations in pigeons. Joumal of Experimental Psychology: Animal Behavior Processes, 14, 401-412.

Balsam, P., Locurto, C. M., Terrace, H. S., \& Gibbon, J. (1980). A search for preexposure effects in autoshaping: Effects of US-only or random CS-US presentations, intertrial interval, and number of pretraining trials. Psychological Record, 30, 561-570.

Balsam, P., SChWARTZ, A. L. (1981). Rapid contextual conditioning in autoshaping. Joumal of Experimental Psychology: Animal Behavior Processes, 7, 382-393.

Bolles, R. C., \& Riley, A. L. (1973). Freezing as an avoidance response: Another look at the operant-respondent distinction. Learning \& Motivation, 4, 268-275.

DA VIS, M. (1970). Effects of interstimulus interval length and variability on startle-response habituation in the rat. Journal of Comparative \& Physiological Psychology, 72, 177-192.

DURlach, P. J. (1982). Pavlovian learning and performance when CS and US are uncorrelated. In M. L. Commons, R. J. Herrnstein, \& A. R. Wagner (Eds.), Quantitative analyses of behavior: Vol. 3. ACquisition (pp. 173-193). Cambridge, MA: Ballinger.

DURLACH, P. J. (1983). Effect of signaling intertrial unconditioned stimuli in autoshaping. Journal of Experimental Psychology: Animal Behavior Processes, 9. 374-389.

EWING, M. F., LAREW, M. B. , Wagner, A. R. (1985). Distributionof-trials effects in Pavlovian conditioning: An apparent involvement of inhibitory backward conditioning with short intertrial intervals. Journal of Experimental Psychology: Animal Behavior Processes, 11, 537-547.

FALK, J. L. (1971). The nature and determinants of adjunctive behavior Physiology \& Behavior, 6, 577-588

Fanselow, M. S. (1981). Naloxone and Pavlovian fear conditioning. Learning \& Motivation, 12, 398-419.

FANSELOW, M. S. (1984). Shock-induced analgesia on the formalin test: Effects of shock severity, naloxone, hypophysectomy and associative variables. Behavioral Neuroscience, 98, 79-95.

FANSELOW, M. S. (1989). The adaptive function of conditioned defensive behavior: An ecological approach to Pavlovian stimulussubstitution theory. In R. J. Blanchard, P. F. Brain, D. C. Blanchard, \& S. Parmigiani (Eds.), Ethoexperimental approaches to the study of behavior (pp. 151-166). Dordrecht, The Netherlands: Kluwer Academic Publishers.

FANSELOW, M. S. (1991). Analgesia as a response to aversive Pavlovian conditional stimuli: Cognitive and emotional mediators. In M. R. Denny (Ed.), Fear, avoidance, and phobias: A fundamental analysis (pp. 6186). Hillsdale, NJ: Erlbaum.

FANSELOW, M. S., \& Bolles, R. C. (1979). Naloxone and shock-elicited freezing. Journal of Comparative \& Physiological Psychology, 93, 736-744.

FANSELOW, M. S., \& LeSTER, L. S. (1988). A functional behavioristic approach to aversively motivated behavior: Predatory imminence as a determinant of the topography of defensive behavior. In R. C. Bolles \& M. D. Beecher (Eds.), Evolution and learning (pp. 185-212). Hillsdale, NJ: Erlbaum.

FAnselow, M. S., \& Tighe, T. J. (1988). Contextual conditioning with massed versus distributed unconditioned stimuli in the absence of explicit conditional stimuli. Joumal of Experimental Psychology: Animal Behavior Processes, 14, 187-199.

Grbon, J., Baldock, M. D., Locurto, C., Gold, L., * Terrace, H. S.
(1977). Trial and intertrial durations in autoshaping. Journal of Experimental Psychology: Animal Behavior Processes, 3, 264-284.

GibBon, J., BALSAM, P. (1981). Spreading association in time. In C. M. Locurto, H. S. Terrace, \& J. Gibbon (Eds.), Autoshaping and conditioning theory (pp. 219-253). New York: Academic Press.

GodDARD, M. J., \& JENKINS, H. M. (1988). Blocking of a CS-US association by a US-US association. Journal of Experimental Psychology: Animal Behavior Processes, 14, 177-186.

Gormezano, I., Kenoe, E. J. (1981). Classical conditioning and the law of contiguity. In P. Harzem \& M. D. Zeiler (Eds.), Predictability, correlation, and contiguiry (pp. 1-45). New York: Wiley.

Gormezano, I., MOORE, J. W. (1969). Classical conditioning. In M. H. Marx (Ed.), Learning: Processes (pp. 121-203). Toronto: McMillan.

GrAU, J. (1987). The central representation of an aversive event maintains the opioid and nonopioid forms of analgesia. Behavioral Neuroscience, 101, 272-288

Hayes, R. L., Bennett, G. J., Newlon, P. G., Mayer, D. J. (1978). Behavioral and physiological studies of non-narcotic analgesia in the rat elicited by certain environmental stimuli. Brain Research, 155, 69-90.

Hineline, P. N. (1977). Negative reinforcement and avoidance. In . W. K. Honig \& J. E. R. Staddon (Eds.), Handbook of operant behavior (pp. 364-431). Englewood Cliffs, NJ: Prentice-Hall.

Holder, M. D., Roberts, S. (1988). Effect of intertrial interval on variable-interval discrete-trial barpressing. Animal Learning \& Behavior, 16, 340-353.

Hurwitz, H. M. B., \& Davis, H. (1983). Depriving rats of food: A reappraisal of two techniques. Joumal of the Experimental Analysis of Behavior, 40, 211-213.

KAPLAN, P. S. (1984). Importance of relative temporal parameters in trace autoshaping: From excitation to inhibition. Joumal of Experimental Psychology: Animal Behavior Processes, 10, 113-126.

Killeen, P. R. (1975). On the temporal control of behavior. Psychological Review, 82, 89-115

KILLEEN, P. R. (1979). Arousal: Its genesis, modulation, and extinction. In M. D. Zeiler \& P. Harzem (Eds.), Advances in the experimental analysis of behavior: Vol. 1. Reinforcement and the organization of behavior (pp. 31-78). Chichester, U.K.: Wiley.

Killeen, P. R., Hanson, S. J., Osborne, S. R. (1979). Arousal: Its genesis and manifestation as response rate. Psychological Review, $85,571-581$.

Longo, N., Klempay, S., Bitterman, M. E. (1964). Classical appetitive conditioning in the pigeon. Psychonomic Science, 1, 19-20.

LYSLE, D. T. . FowLER, H. (1988). Changes in pain reactivity induced by unconditioned and conditioned excitatory and inhibitory stimuli. Journal of Experimental Psychology: Animal Behavior Processes, 14, 376-389.

MACKINTOSH, N. J. (1974). The psychology of animal leaming. London: Academic Press.

MACKINTOSH, N. J. (1983). Conditioning and associative learning. Oxford, U.K.: Clarendon Press.

McAllister, W. R., McAllister, D. E., Weldin, G. H.. * COHEN, J. M. (1974). Intertrial interval effects in classical conditioning of fear to a discrete conditioned stimulus and to situational cues Joumal of Comparative and Physiological Psychology, 87, 582-590.

MILLER, R. R., MATZEL, L. D. (1988). The comparator hypothesis: A response rule for the expression of associations. Psychology of Learning \& Motivation, 22, 51-92.

Miller, R. R., Schachtman, T. R. (1985). Conditioning context as an associative baseline: Implications for response generation and the nature of conditioned inhibition. In R. R. Miller \& N. E. Spear (Eds.), Information processing in animals: Conditioned inhibition (pp. 51-88). Hillsdale, NJ: Erlbaum.

MinOR, T. (1987). Stimulus- and pellet-induced drinking during successive discrimination. Joumal of the Experimental Analysis of Behavior, 48, 61-80.

Papini, M. R., Mustaca, A. E., \& Frasca Ponce, A. (1989). Ap- 
petitive contextual learning in rats: Preliminary tests of scalar expectancy theory. Unpublished manuscript.

Papini, M. R., Mustaca, A. E., Tiscornia, G., \& Di Tella, M. (1987). Context learning in the marsupial Lutreolina crassicaudata. International Journal of Comparative Psychology, 1, 126-134.

Pavlov, I. P. (1927). Conditioned reflexes. Oxford, U.K.: Oxford University Press.

Reid, A. K., Vazquez, P. P., \& Rico, J. A. (1985). Schedule induction and the temporal distributions of adjunctive behavior on periodic water schedules. Animal Learning \& Behavior, 13, 321-326.

Rescorla, R. A., \& Durlach, P. J. (1987). The role of context in intertrial interval effects in autoshaping. Quarterly Journal of Experimental Psychology, 39B, 35-48.

Rescorla, R. A., \& Wagner, A. R. (1972). A theory of Pavlovian conditioning: Variations in the effectiveness of reinforcement and nonreinforcement. In A. H. Black \& W. F. Prokasy (Eds.), Classical conditioning II: Current theory and research (pp. 64-99). New York: Appleton-Century-Crofts.

Riley, A. L., Wetherington, C. L., Delamater, A. R., Peele, D. B., \& DaCANAY, R. J. (1985). The effects of variations in the interpellet interval on wheel running in the rat. Animal Learning \& Behavior, 13, 201-206.

Ross, R. T., \& RANDICH, A. (1985). Associative aspects of conditioned analgesia evoked by a discrete CS. Animal Learning \& Behavior, 13, 419-431.

ShefField, F. D., \& CAMPBell, B. A. (1954). The role of experience in the "spontaneous" activity of hungry rats. Journal of Comparative \& Physiological Psychology, 47, 97-100.

Sidman, M. (1962). Reduction in shock frequency as reinforcement for avoidance behavior. Joumal of the Experimental Analysis of Behavior, 5, 247-257.

SKINNER, B. F. (1948). Superstition in the pigeon. Journal of Experimental Psychology, 38, 168-172.
Solomon, R. L., \& CoRBIT, J. D. (1974). An opponent-process theory of motivation: 1. Temporal dynamics of affect. Psychological Review, 81, 119-145.

STADDON, J. E. R. (1977). Schedule-induced behavior. In W. K. Honig \& J. E. R. Staddon (Eds.), Handbook of operant behavior (pp. 125152). Englewood Cliffs, NJ: Prentice-Hall.

STADDON, J. E. R., \& AYRES, S. (1975). Sequential and temporal properties of behavior induced by a schedule of periodic food delivery. Behaviour, 54, 26-49.

Staddon, J. E. R., \& Simmelhag, V. L. (1971). The "superstition" experiment: A reexamination of its implications for the principles of adaptive behavior. Psychological Review, 78, 3-43.

TOMIE, A., \& ABBONDANDOLO, D. (1981). Effect of pretraining US density and test ITI upon the acquisition of autoshaping. Bulletin of the Psychonomic Society, 17, 123-126.

WAGNER, A. R. (1978). Expectancies and the priming of STM. In S. H. Hulse, H. Fowler, \& W. K. Honig (Eds.), Cognitive processes in animal behavior (pp. 177-209). Hillsdale, NJ: Erlbaum.

WAGNER, A. R. (1981). SOP: A model of automatic memory processing in animal behavior. In N. E. Spear \& R. R. Miller (Eds.), Information processing in animals: Memory mechanisms (pp. 5-47). Hillsdale, NJ: Erlbaum.

WaGNer, A. R., \& LAREW, M. B. (1985). Opponent processes and Pavlovian inhibition. In R. R. Miller \& N. E. Spear (Eds.), Information processing in animals: Conditioned inhibition (pp. 233-265). Hillsdale, NJ: Erlbaum.

ZaMBLE, E. (1967). Classical conditioning of excitement anticipatory to food reward. Journal of Comparative \& Physiological Psychology, 63, 516-529.

(Manuscript received October 26, 1989; revision accepted for publication December 31,1990 .) 